\title{
Detection of Candida Endophthalmitis in a Newborn Using Handheld Spectral-Domain Optical Coherence Tomography
}

\author{
Carlos M. Dulanto-Reinoso Maria Ana Martinez-Castellanos
}

Asociación para Evitar la Ceguera en México, Hospital Dr. Luis Sanchez Bulnes, Mexico, Mexico

\section{Keywords}

Candida $\cdot$ Endophthalmitis $\cdot$ Infant $\cdot$ Newborn $\cdot$ Optical coherence tomography

\begin{abstract}
In a newborn with candidemia, two retinal lesions were seen without external inflammatory signs or reaction in the anterior chamber or vitreous. However, handheld spectral domainoptical coherence tomography (HH SD-OCT) images show that one of the retinal lesions had a "firework display" projecting to the vitreous that was not identified in indirect ophthalmoscopy. This finding suggested a Candida endophthalmitis. HH SD-OCT findings allowed us to make an accurate diagnosis and, therefore, modified the decision-making process in the treatment of the pathology.

(C) 2018 The Author(s)

Published by S. Karger AG, Basel
\end{abstract}

\section{Introduction}

Candida endophthalmitis is an ocular infection that severely threatens vision [1]. It often occurs as a complication of candidemia [2]. Endophthalmitis diagnosis is based on the patient's symptoms and findings that are seen in the ophthalmological examination. However, in newborns or patients who are unable to communicate or complain of ocular discomfort, the diagnosis could be more complicated [3]. Currently, there are multiple imaging tests that allow 
an accurate diagnosis in patients whose assessment is a challenge for the ophthalmologist. To the best of our knowledge, this is the first reported case in newborns in which handheld spectral domain-optical coherence tomography (HH SD-OCT) shows a "firework display" projecting to the vitreous. This finding allowed the diagnosis of endophthalmitis by Candida.

\section{Case Report}

A 2,082-g male infant was born at 36 weeks of gestation. His teenage mother did not have prenatal care, underwent unassisted home birth, and was diagnosed with a urinary tract infection upon admission. The infant was admitted to the neonatal intensive care unit due to acute respiratory distress. Moreover, the patient had the following diagnoses: fungal sepsis with two positive culture for Candida albicans, pneumonia, patent ductus arteriosus, anemia, apnea of prematurity and gastroesophageal reflux disease. He was treated with broad-spectrum antibiotics and amphotericin B. Furthermore, the patient received multiple blood transfusions.

At 27 days of age, the infant's ophthalmological evaluation showed a nonhyperemic conjunctiva without anterior chamber reaction or hypopyon. Furthermore, left eye posterior segment examination revealed two lesions in the retina without vitritis. One of them was a fluffy white lesion near the papilla with ill-defined margins and irregular edges, and the other was a rounded macular lesion (Fig. 1a, b). The rest of the ophthalmological evaluation had no additional significant pathological findings.

Fluorescein angiography, performed by wide-angle retinal images (RetCam II; Clarity Medical Systems, Pleasanton, CA, USA), revealed two hyperfluorescent lesions with ill-defined margins and irregular edges compatible with lesions found in the left eye fundoscopy. Additionally, peripheral vascular incompetence in the temporal retina was observed (Fig. 2a, b). HH SD-OCT (Optovue, Fremont, CA, USA) was done in a nonsedated patient. To minimize the discomfort of the evaluation, pacifiers and oral sucrose were administered. Once a complete dilation of the pupil was obtained, a drop of topical anesthetic agent such as proparacaine was instilled and a palpebral speculum was inserted. After that, hypromellose $2 \%$ was placed to aid to protect the ocular surface. Due to the fact that the patient could not fix the gaze on a point, the HH SD-OCT was placed on the eye that we intended to examine, and several images were taken during 5 min until obtaining an image of good quality. OCT imaging showed a macular lesion with high reflectivity in the inner retina with hyperreflective dots in the vitreous that suggest perilesional focal vitritis. In addition, this lesion causes an indirect hyporeflectivity of the outer retina, the pigmentary epithelium of the retina, and the choroid. OCT image shows that the injury is not only limited to the retina, but also affects the vitreous with vitreous cells as a "firework display." This finding reveals the progression from retinitis to endophthalmitis (Fig. 3).

The final diagnosis was endophthalmitis by $C$. albicans that was confirmed by the isolation of this pathogen in the culture of aqueous humor. The patient was treated with intravitreal voriconazole $50 \mu \mathrm{g} / 0.05 \mathrm{~mL}$ and systemic amphotericin B without resolving the retinal lesions and with an increase in vitritis that hindered the evaluation of the fundus. Due to the torpid evolution of the case, a surgical procedure was indicated; however, vitrectomy could not be done due to high systemic surgical risk. Finally, the patient died because of multiple comorbidities. 


\section{Case Reports in Ophthalmology}

\section{Discussion}

The importance of this case is based on obtaining SD-OCT images when retinal lesions caused by Candida are seen in eye fundus examination in newborns or patients who cannot communicate or complain of eye discomfort. It should be noted that SD-OCT provides important subclinical anatomical information that may go unnoticed in an ophthalmological evaluation of routine [4]. HH SD-OCT made the diagnosis of endophthalmitis.

In our case, the newborn had two blood cultures positive for Candida. It is also important to suspect ocular compromise when a patient has several positive blood cultures for fungi [3]. Dilated retinal examination should be performed in patients with candidemia within the first week of treatment [5]. To our knowledge, for those patients without abnormal findings at baseline examination, follow-up retinal assessments should be done in 2 weeks [3] because $20 \%$ of patients develop ocular involvement by Candida after the first week of treatment [6]. However, new examinations can be performed before that time if the patient complains of new symptoms or is unable to communicate [3].

The ophthalmological assessment revealed two retinal lesions. These findings suggested a retinitis by Candida. Likewise, fluorescein angiography showed peripheral vasculitis, a nonspecific and poorly contributory finding. Because retinal lesions were identified in the fundus examination, HH SD-OCT was performed, a diagnostic procedure that is not commonly done in newborns, to assess the extent of retinal lesions and vitreous involvement. HH SD-OCT images showed a lesion with high reflectivity in the inner retina with a "firework display" projecting to the vitreous [7]. Although the vitreous did not have an inflammatory reaction in the ophthalmological evaluation, this SD-OCT finding is characteristic of early endophthalmitis by Candida. Therefore, this diagnostic tool made the diagnosis of Candida endophthalmitis and allowed doctors to provide an appropriate and timely treatment. It should be mentioned that treatment is based on the location of the eye infection. According to Clinical Practice Guideline for the Management of Candidiasis: 2016 Update by the Infectious Diseases Society of America, a systemic and intravitreal antifungal treatment is indicated for Candida retinitis without vitritis. Unlike when a Candida endophthalmitis or Candida chorioretinitis with vitritis develops, as can be seen on our case images obtained from the HH SD-OCT, an early vitrectomy should be considered in addition to antifungal treatment [5]. Early vitrectomy reduces the risk of retinal detachment [1].

The permanent evolution of imaging technology helps us to identify findings that in some pathologies are not evident in a clinical examination performed by expert pediatric ophthalmologists [8]. Recently, HH SD-OCT has been used mainly for the evaluation of the pediatric retina in cases such as retinopathy of prematurity, suboptimal vision and unexplained vision loss, and nystagmus and night blindness because SD-OCT images give additional anatomical information that cannot be seen in fundus examination [5]. However, its greatest benefit is seen in intraocular tumors and mass lesions in which it improves clinical description of a pathology in up to $90 \%$ of cases [9]. It is important to point out that in newborns it is necessary to configure optical parameters for each patient as this improves image quality [10]. The main advantage we found is that the test can be done without sedation or anesthesia in a newborn. In addition, it allows evaluating mid-periphery of the retina easily by tilting the probe. On the other hand, one of the main disadvantages is that HH SD-OCT images depend on operator skill. Also, HH SD-OCT does not have the eye tracking system that is present on tabletop devices.

HH SD-OCT is a useful tool in retinal evaluation in pediatric patients that can help improve clinical diagnosis and modify disease management. We propose that this ancillary test be 
considered for routine screening in newborns when a vitreoretinal disease is suspected in order to assess retinal lesion and vitreous involvement.

\section{Statement of Ethics}

The authors have no ethical conflicts to disclose.

\section{Disclosure Statement}

The authors have no proprietary interest or research funding.

\section{Financial Support}

None.

\section{References}

1 Sallam A, Taylor SR, Khan A, McCluskey P, Lynn WA, Manku K, et al. Factors determining visual outcome in endogenous Candida endophthalmitis. Retina. 2012 Jun;32(6):1129-34.

2 Oude Lashof AM, Rothova A, Sobel JD, Ruhnke M, Pappas PG, Viscoli C, et al. Ocular manifestations of candidemia. Clin Infect Dis. 2011 Aug;53(3):262-8.

3 Geraymovych E, Conduff JH, Braich PS, Leffler CT, Brar VS. Prevalence and factors predictive of intraocular fungal infection in patients with fungemia at an academic urban tertiary care center. Clin Ophthalmol. 2015 Sep;9:1853-8.

4 Huang LL, Hirose T. Portable optical coherence tomography in management of vitreoretinal diseases: current developments, indications, and implications. Semin Ophthalmol. 2012 Sep-Nov;27(5-6):213-20.

5 Pappas PG, Kauffman CA, Andes DR, Clancy CJ, Marr KA, Ostrosky-Zeichner L, et al. Clinical Practice Guideline for the Management of Candidiasis: 2016 Update by the Infectious Diseases Society of America. Clin Infect Dis. 2016 Feb;62(4):e1-50.

6 Nagao M, Saito T, Doi S, Hotta G, Yamamoto M, Matsumura Y, et al. Clinical characteristics and risk factors of ocular candidiasis. Diagn Microbiol Infect Dis. 2012 Jun;73(2):149-52.

7 McGowan G, Lockington D, Imrie F. The firework display of fungal endogenous endophthalmitis. Postgrad Med J. 2010 Aug;86(1018):509.

8 Lee AC, Maldonado RS, Sarin N, O'Connell RV, Wallace DK, Freedman SF, et al. Macular features from spectral-domain optical coherence tomography as an adjunct to indirect ophthalmoscopy in retinopathy of prematurity. Retina. 2011 Sep;31(8):1470-82.

9 Mallipatna A, Vinekar A, Jayadev C, Dabir S, Sivakumar M, Krishnan N, et al. The use of handheld spectral domain optical coherence tomography in pediatric ophthalmology practice: our experience of 975 infants and children. Indian J Ophthalmol. 2015 Jul;63(7):586-93.

10 Maldonado RS, Izatt JA, Sarin N, Wallace DK, Freedman S, Cotten CM, et al. Optimizing hand-held spectral domain optical coherence tomography imaging for neonates, infants, and children. Invest Ophthalmol Vis Sci. 2010 May;51(5):2678-85. 


\section{Case Reports in Ophthalmology}

\begin{tabular}{l|l}
\hline Case Rep Ophthalmol 2018:9:439-443 \\
\hline DOI: 10.1159/000493679 & $\begin{array}{l}\text { (c) 2018 The Author(s). Published by S. Karger AG, Basel } \\
\text { www.karger.com/cop }\end{array}$ \\
\hline
\end{tabular}

Dulanto-Reinoso and Martinez-Castellanos: Handheld SD-OCT in Candida Endophthalmitis in a Newborn
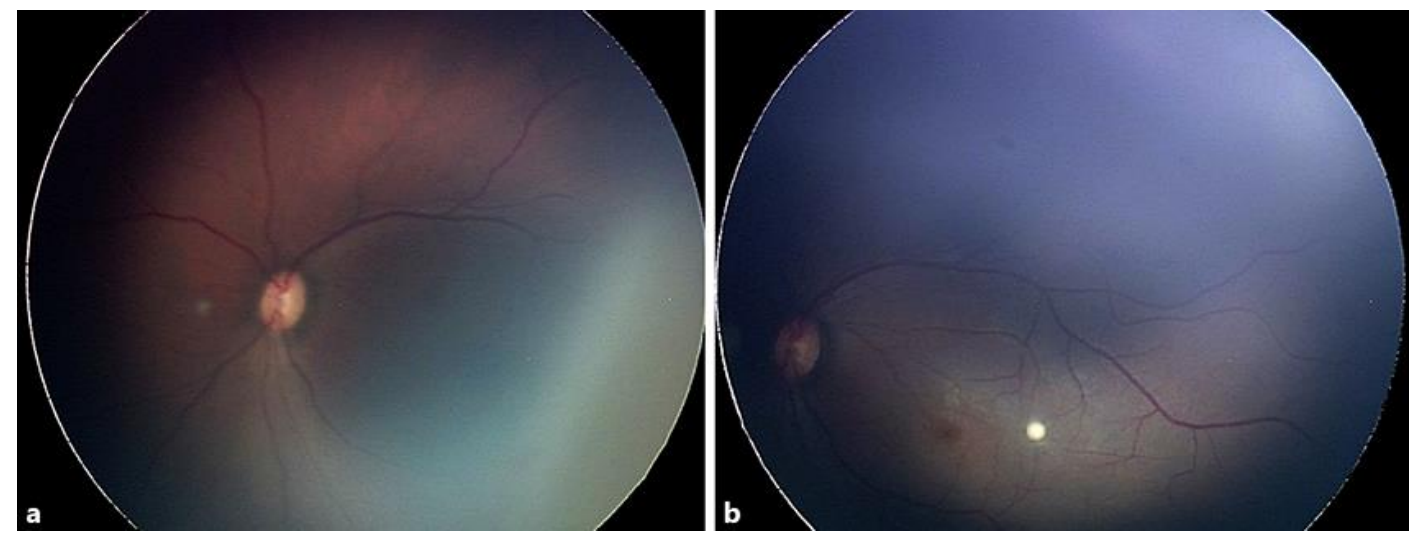

Fig. 1. Left eye fundus photography. Two retinal lesions without vitritis were seen in the left eye fundus evaluation.
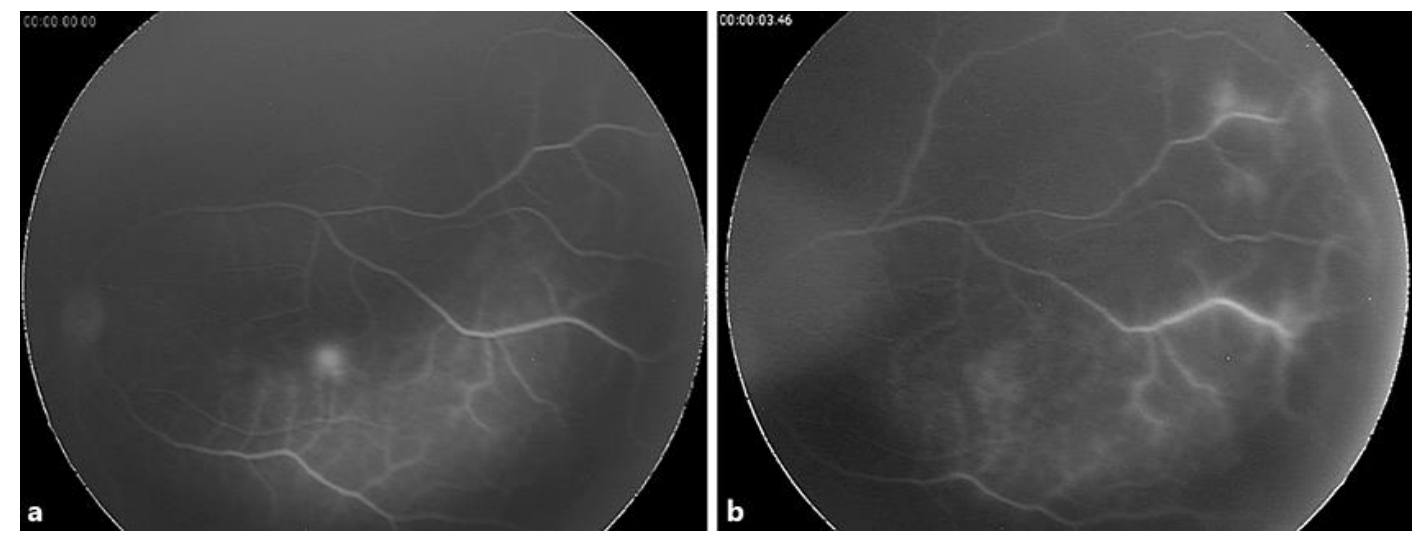

Fig. 2. Left eye fluorescein angiography. Fluorescein angiography, performed by wide-angle retinal images, revealed two hyperfluorescent lesions with ill-defined margins and irregular edges compatible with lesions found in the left eye fundoscopy. Additionally, peripheral vascular incompetence in the temporal retina was observed.

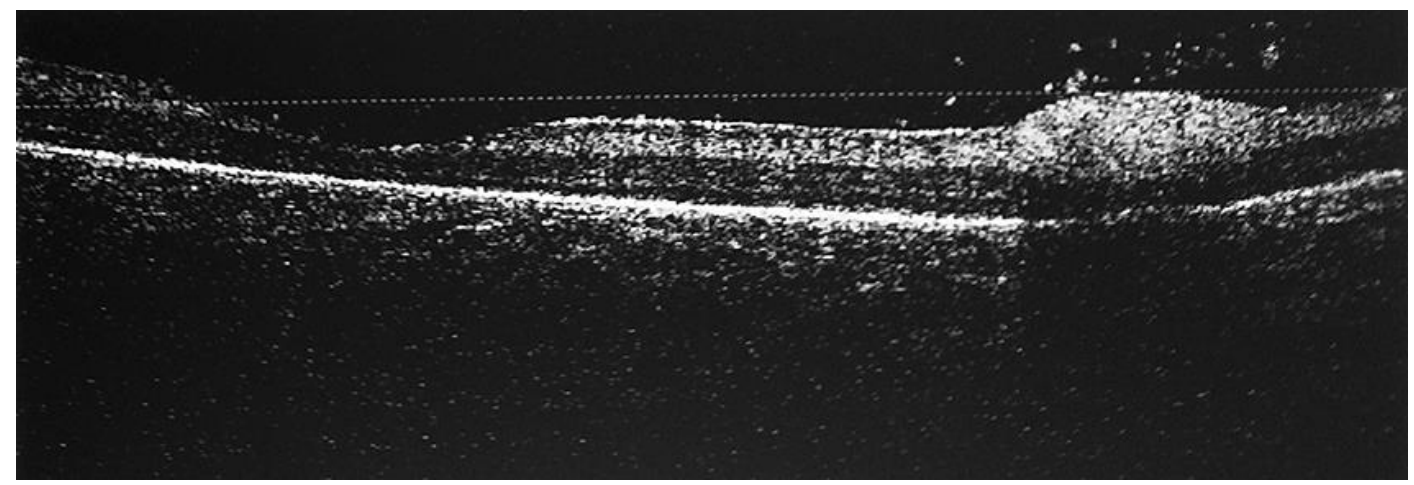

Fig. 3. Left eye optical coherence tomography. A hyperreflective lesion is observed at the level of the outer retina with discrete vitritis in the SD-OCT. 